\title{
When Are the MLSD Respectively the Matched Filter Receiver Optimal with Respect to the BER?
}

\author{
Per Ödling \\ Håkan B. Eriksson ${ }^{1}$ \\ Timo Koski ${ }^{2}$ \\ Per Ola Börjesson ${ }^{1}$ \\ ${ }^{1}$ Division of Signal Processing, Luleå University of Technology, S-971 87 Luleå, Sweden \\ ${ }^{2}$ Department of Mathematics, Royal Institute of Technology, S-100 44 Stockholm, Sweden
}

\begin{abstract}
We reconsider the minimum/optimal bit-error probability receiver (OBER) for intersymbol interference channels with Gaussian noise and the reception of finite blocks of bits. We view the OBER as a function with two inputs: the received sequence and an expected signalto-noise ratio; and one output: the estimated block of bits. Assuming that all sequences are equally probable to be transmitted we prove two results about the behaviour of the OBER. We show that the OBER coincides with the maximum likelihood sequence detector when designed for high signal-to-noise ratios and that it collapses to a matched filter followed by a hard-limiting device for low expected signal-to-noise ratios.
\end{abstract}

\section{A BLOCK TRANSMISSION SYSTEM MODEL}

After the introduction of the Viterbi detector as a Maximum Likelihood Sequence Detector (MLSD) [3], the optimal, or minimum, bit-error probability receiver (OBER) [1], [2], [4] for intersymbol interference (ISI) channels has not been given much attention as a practical receiver. We reconsider the OBER for block transmission systems, to gain insight to its properties and its relation to the MLSD.

Consider the transmission of blocks of binary data through a channel with known ISI and additive Gaussian noise at the receiver. Let the vector $\mathbf{b} \in\{-1,+1\}^{N}$ denote the block of independent bits to be transmitted. We represent the transmission system in matrix notation as:

$$
\mathbf{y}=\mathbf{H b}+\mathbf{n},
$$

where $\mathbf{H}$ is a deterministic and known matrix representing the ISI, the noise $\mathbf{n} \in N\left(\mathbf{0}, \sigma_{\mathbf{n}}^{2} \mathbf{I}\right)$ and $\mathbf{y}$ is the $(N+L) \times 1$ stochastic vector observed by the receiver. Further, let $\boldsymbol{\eta}$ denote the outcome of $\mathbf{y}$.

\section{The Optimal Bit-Error Probability RECEIVER}

Let us consider the detection of $(\mathbf{b})_{\text {bit } k}$. A geometric interpretation of this binary hypothesis testing is that of choosing the correct halfcube:

$$
H_{0}: \quad \mathbf{b} \in \mathcal{C}_{k}^{+}, \quad H_{1}: \quad \mathbf{b} \in \mathcal{C}_{k}^{-},
$$

where $\mathcal{C}_{k}^{+}$and $\mathcal{C}_{k}^{-}$are the halfcubes with (b) bit $k=+1$ and $(\mathbf{b})_{\text {bit } k}=-1$, respectively. The Bayes decision rule minimizing the probability of detection error is given by $[1],[2],[4]$

$$
\Lambda_{k}(\mathbf{y}) \triangleq \frac{f_{\mathbf{y} \mid H_{1}}\left(\boldsymbol{\eta} \mid H_{1}\right)}{f_{\mathbf{y} \mid H_{0}}\left(\boldsymbol{\eta} \mid H_{0}\right)} \underset{H_{0}}{\stackrel{H_{1}}{\gtrless}} \frac{\operatorname{Pr}\left\{H_{0}\right\}}{\operatorname{Pr}\left\{H_{1}\right\}},
$$

where $\operatorname{Pr}\left\{H_{1}\right\}=1-\operatorname{Pr}\left\{H_{0}\right\}$ is the a priori probability that $H_{1}$ is true, and $f_{\mathbf{y} \mid H_{0}}(\cdot)$ and $f_{\mathbf{y} \mid H_{1}}(\cdot)$ designate the probability density functions for $\mathbf{y}$ given $H_{0}$ and $H_{1}$, respectively.
Proposition 1: Let $\psi(\mathbf{y} \mid \boldsymbol{\beta})$ denote the conditional density of $\mathbf{y}$ given that the sequence $\boldsymbol{\beta}$ was transmitted (here multi-dimensional Gaussian). Furthermore, let

$$
\widehat{\mathbf{b}}_{\mathrm{OBER}}(\mathbf{y}) \triangleq \Gamma\left(\mathbf{y}, \sigma_{\mathbf{n}}\right) \triangleq \operatorname{sign}\left(\sum_{\boldsymbol{\beta} \in \mathcal{C}_{1}^{+}} w(\mathbf{y}, \boldsymbol{\beta}) \boldsymbol{\beta}\right)
$$

and $w(\mathbf{y}, \boldsymbol{\beta})=\psi(\mathbf{y} \mid \boldsymbol{\beta}) \operatorname{Pr}\{\mathbf{b}=\boldsymbol{\beta}\}-\psi(\mathbf{y} \mid-\boldsymbol{\beta}) \operatorname{Pr}\{\mathbf{b}=$ $-\boldsymbol{\beta}\}$, where $\operatorname{Pr}\{\mathbf{b}=\boldsymbol{\beta}\}$ is the probability for the sequence $\boldsymbol{\beta} \in \mathcal{C}$ being transmitted. Then $\widehat{\mathbf{b}}_{\mathrm{OBER}}(\mathbf{y})$ is the detector of the transmitted bits that minimizes the bit-error probability.

Note that (4) represents a parallel block processor structure, simultaneously detecting all the individual bits.

As indicated by (4), we find it instructive to view the OBER as a function $\Gamma(\mathbf{y}, \alpha)$ with two inputs, $\mathbf{y}$ and $\alpha$. The parameter $\alpha^{2}$ is the variance the OBER is designed for, and controls the decision regions in $\mathbb{R}^{N+L}$ where $\mathbf{y}$ takes its values. Thus, the OBER depends on the expected SNR and is only optimal when the expected variance $\alpha^{2}$ and the true variance $\sigma_{\mathbf{n}}^{2}$ agree.

\section{THE BEHAVIOUR OF THE OBER}

We will discuss asymptotical properties of the OBER by studying the function $\Gamma(\cdot, \cdot)$ as defined in (4). Assuming that all sequences are equally probable to be transmitted we show that

$$
\lim _{\alpha \rightarrow 0} \Gamma(\mathbf{x}, \alpha)=\widehat{\mathbf{b}}_{\mathrm{MLSD}}(\mathbf{x}), \quad \text { for all } \mathbf{x} \in \mathbb{R}^{N+L},
$$

and that

$$
\lim _{\alpha \rightarrow \infty} \Gamma(\mathbf{x}, \alpha)=\operatorname{sign}\left(\mathbf{H}^{T} \mathbf{x}\right), \quad \text { for all } \mathbf{x} \in \mathbb{R}^{N+L} .
$$

Equation (5) means that the OBER designed for a high SNR becomes the MLSD. It is because of this is that the MLSD will achieve the minimum attainable bit-error probability when used in systems with a high SNR, $c f$. [3]. In equation (6) we find a similar comparison for low SNR between the OBER and the matched filter with hard decisions. If the true SNR is low, the best possible receiver is actually the matched filter receiver as comes to the BER.

\section{REFERENCES}

[1] K. Abend, T.J. Harley and B.D. Fritchman, "On optimum receivers for channels having memory," IEEE Trans. Inf. Th., vol. 14 , no. 6 , pp. 818-820, November 1968.

[2] R.R. Bowen, "Bayesian decision procedure for interfering digital signals," IEEE Trans. Inf. Th., vol. 15, no. 4, pp. 506-507, July 1969.

[3] D.G. Forney, "Maximum likelihood sequence estimation of digital sequences in the presence of intersymbol interference," IEEE Trans. Inf. Th., vol. 18, no. 3, pp. 363-378, May 1972.

[4] P. Ödling, H.B. Eriksson, T. Koski and P.O. Börjesson, "Representations for the minimum bit-error probability receiver for block transmission systems with intersymbol interference channels," Research Report 1995:11, Luleå University of Technology, Luleå, Sweden, April 1995. 